University of Nebraska - Lincoln

DigitalCommons@University of Nebraska - Lincoln

Faculty Publications from Nebraska Center for

Materials and Nanoscience

Materials and Nanoscience, Nebraska Center

for (NCMN)

$5-15-2002$

\title{
Magnetization reversal of individual nanowires with controlled defects
}

Andrei Sokolov

University of Nebraska-Lincoln, sokolov@unl.edu

Renat F. Sabirianov

University of Nebraska at Omaha, rsabirianov@mail.unomaha.edu

W. Wernsdorfer

Laboratoire de Magnetisme Louis Neel, CNRS, BP166, 38042 Grenoble Cedex 9, France

Bernard Doudin

University of Nebraska-Lincoln, bernard.doudin@ipcms.unistra.fr

Follow this and additional works at: https://digitalcommons.unl.edu/cmrafacpub

Part of the Nanoscience and Nanotechnology Commons

Sokolov, Andrei; Sabirianov, Renat F.; Wernsdorfer, W.; and Doudin, Bernard, "Magnetization reversal of individual nanowires with controlled defects" (2002). Faculty Publications from Nebraska Center for Materials and Nanoscience. 37.

https://digitalcommons.unl.edu/cmrafacpub/37

This Article is brought to you for free and open access by the Materials and Nanoscience, Nebraska Center for (NCMN) at DigitalCommons@University of Nebraska - Lincoln. It has been accepted for inclusion in Faculty Publications from Nebraska Center for Materials and Nanoscience by an authorized administrator of DigitalCommons@University of Nebraska - Lincoln. 


\title{
Magnetization reversal of individual nanowires with controlled defects
}

\author{
A. Sokolov a) and R. Sabirianov \\ Department of Physics and Astronomy and Center for Materials Research and Analysis, Behlen Laboratory \\ of Physics, University of Nebraska, Lincoln, Nebraska 68588-0111 \\ W. Wernsdorfer \\ Laboratoire de Magnetisme Louis Neel, CNRS, BP166, 38042 Grenoble Cedex 9, France \\ B. Doudin \\ Department of Physics and Astronomy and Center for Materials Research and Analysis, Behlen Laboratory \\ of Physics, University of Nebraska, Lincoln, Nebraska 68588-0111
}

Low temperature magnetization reversal measurements were performed on individual permalloy nanowires of diameters between 30 and $60 \mathrm{~nm}$. During the electrochemical growth of the wire, a defect was induced by a short pulse in the deposition potential modifying locally the microstructure and composition. Magnetic measurements performed with micro-superconducting quantum interference devices were performed. The angular dependence of switching field revealed significant deviations from classical predictions. For specific angles, magnetization curves indicate a reversal occurring in two steps. (C) 2002 American Institute of Physics.

[DOI: $10.1063 / 1.1452253$ ]

Considerable progress in fabrication of nanoscale magnetic materials stimulates researchers to investigate the underlying mechanisms for magnetization reversal, a crucial point for technical applications. Although the magnetization reversal of such particles had been described back to late 1940 's by Stoner and Wohlfarth ${ }^{1}$ and Néel, ${ }^{2}$ only recently have experimental studies of individual particles become possible with techniques employing magnetic force microscopy, ${ }^{3}$ micro-Hall probes magnetometry, ${ }^{4,5}$ and planar microscale superconducting quantum interference devices ( $\mu$-SQUID) ${ }^{6}$ There are only a few reports in the literature that clearly evidence magnetization reversal following the predictions of Stoner-Wolfarth for lowest temperatures and Néel-Brown theory ${ }^{2,7}$ for thermally activated switching. ${ }^{6,8}, 9$ Very often, the magnetization reversal proceeds through complicated process of curling and domain wall motion. ${ }^{10,11}$

Deviations from the classical simplest predictions are commonly attributed to the presence of defects, shape, particle size and surface roughness. The defect can be characterized by the change in exchange coupling strength, anisotropy (magnitude and direction) and lateral dimensions. For example, Lorentz imaging ${ }^{12}$ revealed that the arrow end of a $\mathrm{NiFe} 200 \mathrm{~nm}$ wide element suppresses the formation of domains and increases the switching field. Injection of a magnetic wall from the modified end of submicron sized magnetic wires was demonstrated by magnetoresistance effects. ${ }^{13}$

The purpose of our work is to attempt to study systems with an artificial defect and to show how the magnetization reversal properties are affected. We study sub-100 nm wires, ideal from the point of view of shape anisotropy. Permalloy minimizes the influence of magnetocrystalline anisotropy and magnetostriction. Wires of diameters of 30-60 nm were prepared by electrochemical deposition inside nanoporous membranes. ${ }^{14}$ We take advantage of the literature on permal-

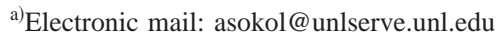

loy electroplating ${ }^{15}$ to reproduce the recipes and successfully make permalloy wires. ${ }^{16}$ Element analysis by electron microscopy techniques showed the $\mathrm{Ni}_{82} \mathrm{Fe}_{18}$ composition within $2 \%$, except for the first $200 \mathrm{~nm}$ of deposition. During the growth, a short pulse in the deposition potential induced a defect, locally modifying the microstructure and composition of NiFe. The position of the defect was chosen to be at the end of the wires synthesis. Membranes were then dissolved, and a low density of wires was deposited on the $\mu$-SQUIDs chips. Superconducting loops with a single magnetic wire of lengths ranging from 2 to $6 \mu \mathrm{m}$ were investigated at a temperature of $50 \mathrm{mK}$. The diameter of the wires was of 45 $\pm 15 \mathrm{~nm}$. This value was deduced from statistics of wire diameters from the same batch of samples, observed by transmission electron microscopy. More detailed descriptions and pictures of similar samples can be found in our preceding works. ${ }^{6,17}$ Here we notice, however, that measured wires remain in a thin polymer covering, which prevents them from oxidation, but causes an embarrassing precise diameter estimation.

Extensive angular measurements were performed. Figure 1 shows the angular dependence of normalized switching field. Shapes of the asteroids indicate that a curling mode appears when the applied field direction deviates from the $z$ axis of wires, as already shown for Ni wires. ${ }^{6}$ For wires of a larger diameter, the polar plot of the switching field becomes more elongated, as expected from a curling model. Figure 1 also shows that the astroid lacks axial symmetry. For a given angle $\alpha$ between the wire axis and applied field, the measured switching field is larger when $\alpha$ exceeds $\pi / 2$, conventionally named "positive" angle in the following discussion. The difference in the magnetization process between positive and negative angles is clearly seen from the magnetization curves of sample $a$., taken for the angles with small positive and negative deviations from the direction normal to the wire axis (Fig. 2). For negative directions, only one jump is ob- 


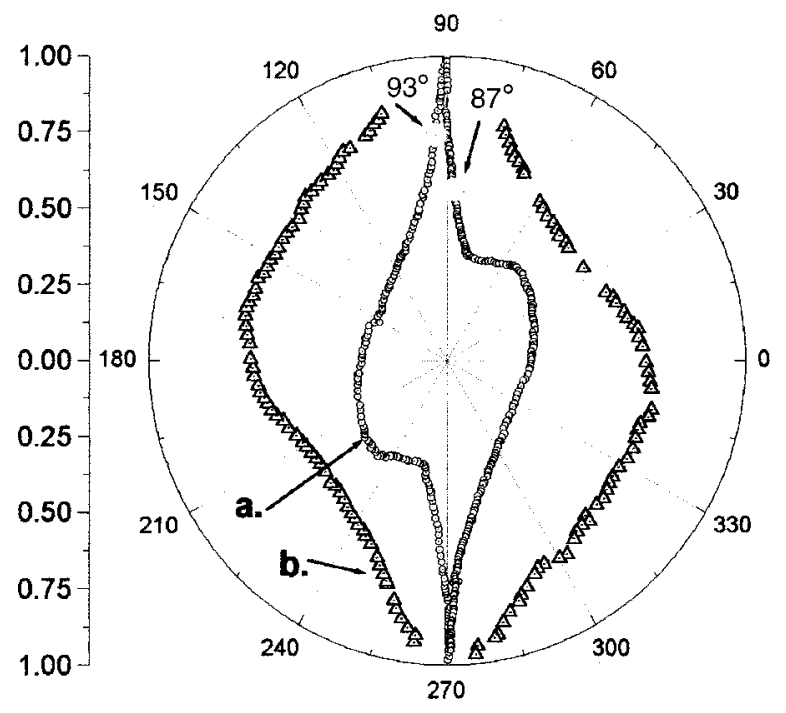

FIG. 1. Angular dependence of switching field for wires of two different diameters $(45 \pm 15 \mathrm{~nm})$. Both curves are normalized to the same maximum value of applied magnetic field $(0.41 \mathrm{~T})$.

served. However, a multiple-step reversal appears at small positive angles. This difference gradually disappears with increasing angle.

In the following, we use the simplest model for understanding the lack of axial symmetry and the occurrence of a two-step reversal for a limited angular window. We use micromagnetic simulations, performed using the Landau-Lifshitz ${ }^{18}$ equation

$$
\frac{d M}{d t}=-\gamma M \times H_{\mathrm{eff}}-\frac{\gamma \alpha}{M} M \times\left(M \times H_{\mathrm{eff}}\right),
$$

where $M$ is the point-wise magnetization, $H_{\text {eff }}$ is the pointwise effective field, $\gamma$ is the gyromagnetic ratio, and $\alpha$ is the damping coefficient. The effective field is defined as $H_{\text {eff }}$ $=-\mu_{0}^{-1} \partial E / \partial M$. The energy density $E$ is a function of $M$ as specified by Brown's equations, and includes crystalline an-

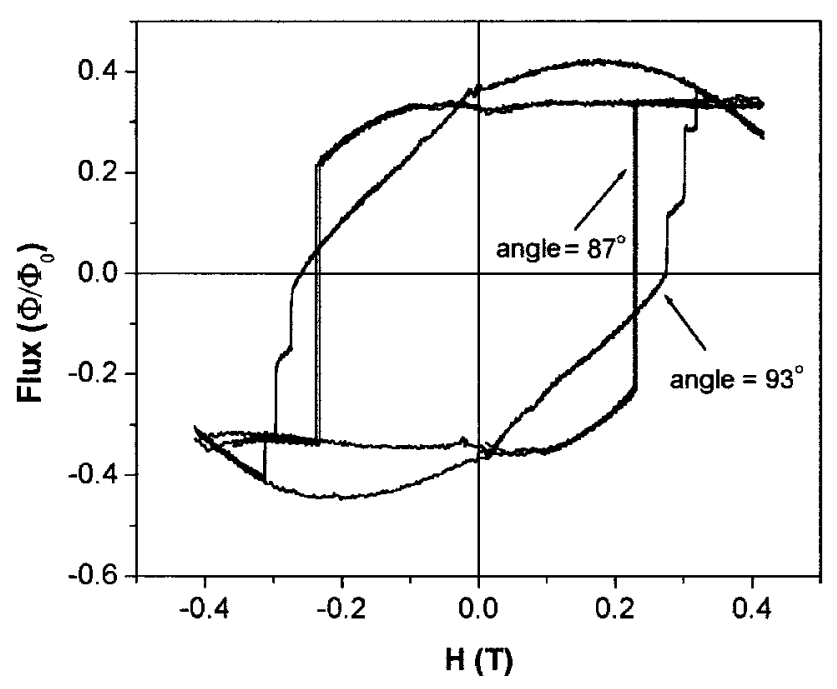

FIG. 2. Magnetization curves for sample a, at two different angles. A singlestep process occurs when the angle is smaller than $90^{\circ}$, and a multiple-step process occurs at angles larger than $90^{\circ}$.

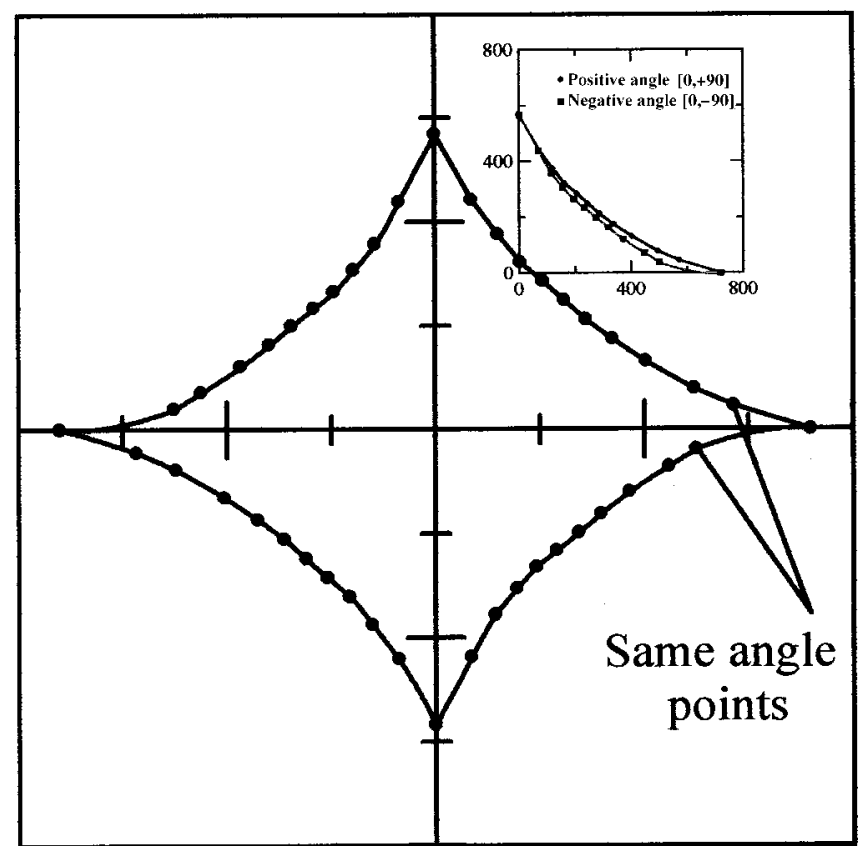

FIG. 3. Calculated Stoner-Wohlfarth asteroid for a wire with a defect at its end. Inset shows more clearly the difference between positive and negative angles.

isotropy, exchange, magnetostatic, and applied field (Zeeman) terms. ${ }^{19}$ The OOMMF micromagnetic software developed at the National Institute for Standards and Technology was used. ${ }^{20}$

We propose using a very thin wire, to simplify our calculations, as well as geometrical considerations, by avoiding the curling model. In the case of an ideal wire $(2.5 \mathrm{~nm}$ $\times 400 \mathrm{~nm}$ ) without induced defect, a nucleation-type process of the magnetization reversal is found. If the wire is magnetized along the $z$ direction and the applied field is growing in the opposite direction, the magnetization reversal nucleates at the ends of the wire, in agreement with experiment. ${ }^{12} \mathrm{Be}-$ cause both ends are equivalent, there is complete axial symmetry of the Stoner-Wohlfarth asteroid.

Let us consider the reversal of the wire when the top and bottom ends are not equivalent. More precisely, the exchange stiffness constant of the top end is reduced from 13 to 5 $\times 10^{-12} \mathrm{~J} / \mathrm{m}$, and the anisotropy constant is reduced from 100 to $-50 \mathrm{~J} / \mathrm{m}^{3}$, i.e., in-plane anisotropy. Furthermore, the anisotropy of the top defect is directed along the (011) direction or $45^{\circ}$ from the axis of the wire. A change of the parameters can originate from surface effects, or more probably from a different composition of the alloy at one end of the wire. Note that this parameter choice of is not critical for the conclusions presented next.

The calculated astroid has lost the axial symmetry in qualitative agreement with experiment (Fig. 3). The difference with experiment is due to the diameter of the real wire, which exceeds $20 \mathrm{~nm}$. In this case, curling-type modes will appear in the micromagnetic simulation. Nevertheless, the main features can be seen in the thin wire model. The switching field for the positive and negative angles are different. The discrepancy in the angular dependence of switching for clockwise and counter-clockwise changes of the applied field 
is clearly seen in the inset in Fig. 3. This difference increases with increasing angle between the applied field and the wire. At positive angles, the nucleus is not formed at the top end because it is hampered by its anisotropy and the reversal is triggered at the bottom end.

Magnetization reversal occurring in a multiple-step process is explained in terms of domain wall pinning at a defect in the middle of the wire. The two sides of the wire switch at different fields, as previously discussed. At positive angles (lowest switching field values), the magnetization reversal is blocked at an impurity site, and a large applied field is necessary to complete the reversal. Our experimental data do not allow us to discriminate between a process of unpinning of a domain wall and a nucleation originating from the other end of the wire. In principle, comparing the second step value with the step occurring at an equivalent negative angle should give the answer. Micromagnetic calculations will allow us to retrieve both scenarios by choosing adequate anisotropy and exchange constants of the defect and end of the sample. At negative angles, the magnetization reversal occurring at a larger field is not blocked at an impurity site.

Our experiments confirm that the two main defects of our permalloy wires are their ends. Furthermore, one end of the wire has a modified composition, which was an intrinsic characteristic of the synthesis method. This asymmetry is reflected in a Stoner-Wohlfarth astroid. The occurrence of a defect along the wire allows the reversal to be pinned, but for a limited angular window. We used micromagnetic calculations, with the minimum hypothesis input, to explain qualitatively our results.
The support of the NSF-INT Program (Grant No. INT 9980705), NSF CAREER Program (Grant No. DMR 9874657), and the Office of Naval Research are gratefully acknowledged.

${ }^{1}$ E. C. Stoner and E. P. Wohlfarth, Philos. Trans. R. Soc. London, Ser. A 240, 599 (1948).

${ }^{2}$ L. Néel, Ann. Geophys. (C.N.R.S.) 5, 99 (1949).

${ }^{3}$ M. Lederman, S. Schultz, and M. Ozaki, Phys. Rev. Lett. 73, 1986 (1984).

${ }^{4}$ A. D. Kent, S. von Mólnar, S. Gilder, and D. D. Awschalom, J. Appl. Phys. 76, 6656 (1994).

${ }^{5}$ J. G. S. Lok, A. K. Geim, J. C. Maan, S. V. Dubonos, L. T. Kuhn, and P. E. Lindelof, Phys. Rev. B 58, 12201 (1998).

${ }^{6}$ W. Wernsdorfer, B. Doudin, D. Mailly, K. Hasselbach, A. Benoit, J. Meier, J.-P. Ansermet, and B. Barbara, Phys. Rev. Lett. 77, 1873 (1996).

${ }^{7}$ W. F. Brown, Phys. Rev. 130, 1677 (1963).

${ }^{8}$ W. Wernsdorfer, E. Bonet Orozco, K. Hasselbach, A. Benoit, B. Barbara, D. Mailly, N. Demoncy, A. Loiseau, and H. Pascard, Phys. Rev. Lett. 78, 4014 (1977).

${ }^{9}$ E. Bonet, W. Wernsdorfer, B. Barbara, A. Benoit, D. Mailly, and A. Thiaville, Phys. Rev. Lett. 83, 4188 (1999).

${ }^{10}$ W. F. Brown, Phys. Rev. 105, 1479 (1957).

${ }^{11}$ A. Aharoni, J. Appl. Phys. 82, 1281 (1997).

${ }^{12}$ T. Schrefl, J. Fidler, K. J. Kirk, and J. N. Chapman, J. Magn. Magn. Mater. 175, 193 (1997).

${ }^{13}$ K. Shigeto, T. Shinjo, and T. Ono, Appl. Phys. Lett. 75, 2815 (1999).

${ }^{14}$ C. R. Martin, Science 266, 1961 (1994).

${ }^{15}$ L. T. Romankiw and J. D. Olsen, in Proceedings of the Second International Symposium on Magnetic Materials, Processes and Devices, edited by L. T. Romankiw and D. A. Herman (Electrochemical Society, Pennington, NJ, 1992), p. 339.

${ }^{16}$ J. Meier, B. Doudin, and J.-P. Ansermet, J. Appl. Phys. 79, 6010 (1996).

${ }^{17}$ W. Wernsdorfer, K. Hasselbach, A. Benoit, B. Doudin, D. Mailly, J. Meier, J.-P. Ansermet, and B. Barbara, Phys. Rev. B 55, 11552 (1997).

${ }^{18}$ L. D. Landau and E. M. Lifshitz, Phys. Z. Sowjetunion 8, 153 (1935).

${ }^{19}$ W. F. Brown, J. Micromagnetics (Krieger, New York, 1978).

${ }^{20} \mathrm{http}:$ \www.nist.govloommf, and links therein. 